\title{
Hydrogels with a macroscopic-scale liquid crystal structure by self-assembly of a semi-rigid polyion complex
}

\author{
Zii Liang $\mathrm{Wu}^{1}$, Takayuki Kurokawa ${ }^{2,3}$ and Jian Ping Gong ${ }^{2,3}$
}

Designing hydrogels with self-assembled or self-organized structures has become an attractive field of research because these hydrogels usually have robust functions and promising applications, such as in artificial tissues and optical sensors. However, the self-organized structures developed in synthetic hydrogels via molecular self-assembly are generally limited to the submicrometer or micrometer level, which is far from the related scale achieved in biological tissues. Therefore, it is desirable to create macroscopically ordered structures in hydrogels; these structures should greatly improve the material's functionalities, such as their optical properties. In this review, we generally introduce our recent studies on the synthesis of hydrogels with

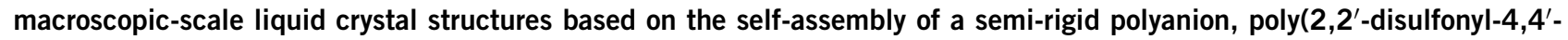
benzidine terephthalamide) (PBDT). Upon electrostatic interaction with multivalent cations or polycations, PBDT molecules form semi-rigid complexes or mesoscopic bundles that further self-assemble into macroscopic organized structures and are frozen by the subsequent gelation process. We have developed physical hydrogels with centimeter-scale anisotropic structures, polycationic hydrogels with millimeter-scale cylindrically symmetric structures and plate gels with cubic-packed concentric domains. This work should contribute to the development of macroscopic self-organized structures in hydrogel materials with specific functions.

Polymer Journal (2012) 44, 503-511; doi:10.1038/pj.2012.74; published online 25 April 2012

Keywords: hydrogels; liquid crystal; macroscopic structure; polyion complex; self-assembly; semi-rigid polyanion

\section{INTRODUCTION}

Nature produces sophisticated structural materials by self-organizing discrete components into ordered and hierarchical structures that follow a well-defined pattern. ${ }^{1-5}$ These self-organized structures of various scales endow the corresponding materials, especially biological systems, with robust additional functions. For example, phospholipids form the lipid bilayer around cell that controls the diffusion of ions, proteins, and so on. ${ }^{1}$ Tropocollagens self-assemble into collagen fibrils that are closely packed into parallel arrays in the tendon, rendering the tendon excellent mechanical properties. ${ }^{1,3}$ Myosin shows a liquid crystal (LC) structure in sarcomeres, which contribute to the formation and smooth motion of muscle fibers. ${ }^{5}$ In these biological tissues, biomacromolecules such as filamentous actins (F-actins) and microtubules with negative charges and rigid or semi-rigid structures significantly contribute to the formation of advanced architectures; this self-assembly of biomacromolecules usually involves multivalent cations, cationic proteins, or liposomes via an electrostatic interaction. $^{6-9}$

Inspired by nature, self-assembly has received widespread attention as a method for developing materials with self-organized structures. ${ }^{10-12}$
These efforts have succeeded in designing various functional materials based on the formation of self-assembled structures in solutions, elastomers, gels and hard materials. ${ }^{12-16}$ For example, block copolymers form micelles in solutions and serve as a vehicle for enhanced drug delivery. Within these self-organized materials, structured hydrogels are of particular significance because they possess properties similar to biological soft tissue (both are soft and wet) and therefore have important potential applications in artificial tissues and optical sensing. ${ }^{16-21}$ During these attempts, biomacromolecules, block copolymers and LC molecules are usually used to develop selforganized structures in synthetic physical or chemical hydrogels via intermolecular noncovalent interactions, such as ionic bonding and hydrophobic interactions. ${ }^{19-30}$ In our previous work, LC hydrogels were synthesized by copolymerization of acrylic acid with LC 11-( $4^{\prime}-$ cyanobiphenyloxy) undecyl acrylate; the gels thus obtained show anisotropic shrinkage as the temperature increases. ${ }^{28,29}$ In addition, Kang et al. ${ }^{25}$ have created a photonic hydrogel with a periodic lamellar structure by using a block copolymer.

The anisotropic structures in hydrogels developed in the former works are generally at the sub-micrometer or micrometer scale. ${ }^{19-29}$

${ }^{1}$ Division of Biological Sciences, Graduate School of Science, Hokkaido University, Sapporo, Japan; ${ }^{2}$ Faculty of Advanced Life Science, Graduate School of Science, Hokkaido University, Sapporo, Japan and ${ }^{3}$ Creative Research Initiative Sousei, Hokkaido University, Sapporo, Japan

Correspondence: Dr JP Gong, Faculty of Advanced Life Science, Graduate School of Science, Hokkaido University, Sapporo, Japan.

E-mail: gong@mail.sci.hokudai.ac.jp

Received 6 February 2012; revised 21 March 2012; accepted 22 March 2012; published online 25 April 2012 
The integrated materials are still macroscopically isotropic because the small self-assembled aggregates usually lack long-range orientation. Using specific substrates or electric, magnetic and shear fields, however, LC and amphiphilic molecules can form macroscopically anisotropic structures that are subsequently frozen in hydrogels. ${ }^{29-35}$ For example, our group has developed a polyacrylamide hydrogel with an immobile macroscopic (single-domain) lamellar structure of poly(dodecylglyceryl itaconate) by applying a shear flow to the precursor solution before polymerization. ${ }^{33-35}$ These poly (dodecylglyceryl itaconate)/polyacrylamide hydrogels show multiple functions, such as reversible and mechanically tunable structural color, one-dimensional swelling and extraordinary toughness. With these external fields, macroscopically anisotropic structures can be developed in hydrogels by controlled molecular self-assembly or by self-assembly via long-range noncovalent interactions. However, thus obtained hydrogels with macroscopic self-organized structures have rarely been reported.

In recent years, one of the systems we have examined is the semirigid polyanion poly $\left(2,2^{\prime}\right.$-disulfonyl- $4,4^{\prime}$-benzidine terephthalamide $)$ (PBDT) and its self-assembling behavior in aqueous solution and hydrogels. ${ }^{36-46}$ We have developed hydrogels with mesoscopic network-like structures via viscoelastic phase separation and millimeter-scale to centimeter-scale ordered structures based on the self-assembly of the semi-rigid polyion complex. ${ }^{38-47}$ In this focused review, we will briefly introduce macroscopically structured hydrogels that are developed by two different methods. Centimeter-scale anisotropic structures are created in a physical hydrogel by dialyzing PBDT aqueous solution in a $\mathrm{CaCl}_{2}$ solution, in which $\mathrm{Ca}^{2+}$ behaves as a physical cross-linker. ${ }^{40,45}$ The diffusion of $\mathrm{Ca}^{2+}$ induces the molecular orientation and electrostatic complexation of the negatively charged PBDT, which forms macroscopically self-assembled structures in the physical hydrogel. By contrast, hydrogels with millimeter-scale anisotropic domains are obtained by polymerizing a cationic monomer in the presence of a small amount of semi-rigid polyanion PBDT, which is used as a dopant. ${ }^{41-44}$ Semi-rigid polyion complexes form during the polymerization and self-assemble into millimeter-scale ordered structures that are frozen by the subsequent chemically cross-linking process. These macroscopically structured hydrogels with good optical properties and sensitive responses to external force may find applications in optical devices and mechano-optical sensors. We believe that this work will promote the development of macroscopically self-organized materials with complex structures and robust functions.

\section{SYNTHESIS OF PHYSICAL HYDROGELS WITH CENTIMETER- SCALE ANISOTROPIC STRUCTURES}

\section{Characteristics of semi-rigid polyanion PBDT}

Before introducing self-organized hydrogels, we would like to discuss some characteristics of PBDT. PBDT, a water soluble polyanion, is synthesized by an interfacial polycondensation reaction. ${ }^{47,48}$ It has a high molecular weight, negative charge and semi-rigid structure, similar to certain natural biomacromolecules, such as DNA and F-actin. The chemical structure of PBDT is shown in Scheme 1a. Aqueous PBDT solutions with different PBDT concentration, $C_{\mathrm{B}}$ are prepared by dissolving a prescribed amount of PBDT in pure water. These PBDT solutions have notably low critical concentrations of nematic LC, $C_{\mathrm{LC}}{ }^{*}$. Figures $1 \mathrm{a}$, b shows polarizing optical microscope (POM) images of PBDT solutions at two different $C_{\mathrm{P}}$. In this part, the PBDT we used has weight-average molecular weight $M \mathrm{w}$ of $1.8 \times 10^{6} \mathrm{~g} \mathrm{~mol}^{-1}$ and $C_{\mathrm{LC}}{ }^{*}$ of $2.2 \mathrm{wt} \%{ }^{41-44}$ When $C_{\mathrm{P}} \geq C_{\mathrm{LC}}{ }^{*}$, a strong birefringence and the schlieren texture of the nematic LC phase can be observed (Figure 1b). ${ }^{37}$ In salt-free or $\mathrm{NaCl}$ aqueous solutions, the PBDT molecules exhibit various self-assembled structures, ranging from cluster-like isotropic assemblies to anisotropic fibers at a submicrometer scale. We will not discuss this property in detail here.

Owing to its high molecular weight and semi-rigid nature, PBDT is easy to align under a mechanical shearing field. ${ }^{36,49}$ Without shearing, the POM image of a PBDT solution is completely dark towing to the relatively low $C_{\mathrm{B}} 2 \mathrm{wt} \%<C_{\mathrm{LC}}{ }^{*}$ (Figure 1a). When a circular shear is applied, however, the image shows strong birefringence (Figure 1c). As the birefringence in Figure 1c is the first white-gray color, this optical property of PBDT can be recognized simply by inserting a 530-nm tint plate. As shown in Figure 1d, a PBDT solution shows blue (additive) and orange (subtractive) colors when oriented in the
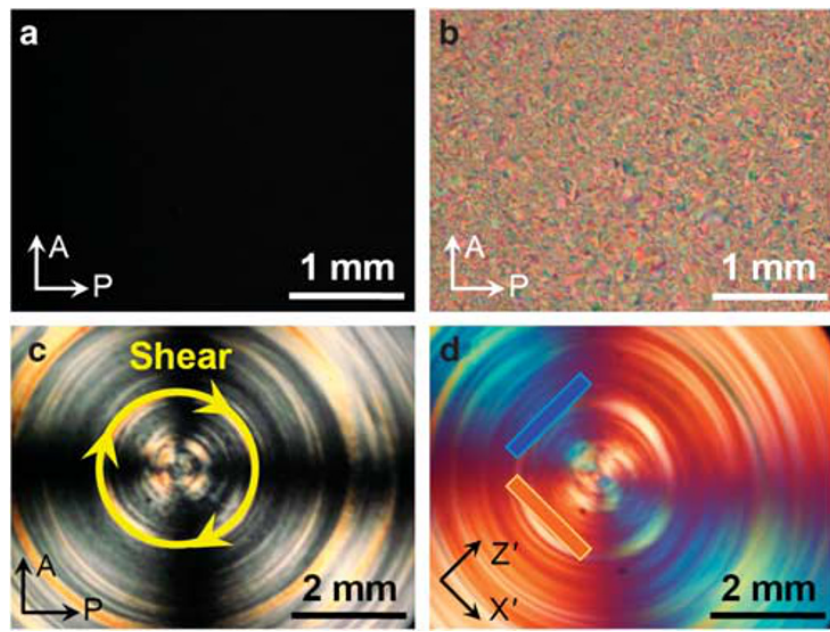

Figure 1 (a, b) POM images of aqueous PBDT solutions with different concentrations of PBDT, $C_{\mathrm{P}}$. (a) $C_{\mathrm{P}}=2$ wt $\%$; (b) $C_{\mathrm{P}}=3$ wt\%. (c, d) Images of a 2-wt\% PBDT aqueous solution under circular shearing observed under POM (c) and after inserting a 530-nm tint plate (d). The $C_{\mathrm{LC}}$ * of the PBDT used is $2.2 \mathrm{wt} \%$. A, the analyzer; $P$, the polarizer; $X^{\prime}$, the fast axis of the tint plate; $Z^{\prime}$, the slow axis of the tint plate.

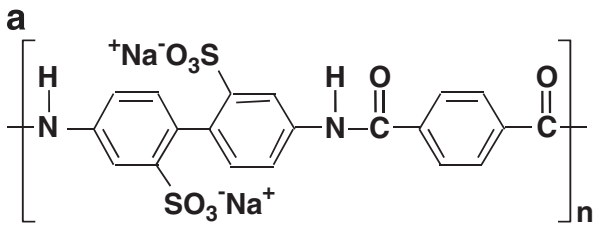

PBDT b<smiles>C=CC(=O)NCCCC</smiles>

DMAPAA-Q

Scheme 1 The chemical structures of (a) the semi-rigid polyanion, PBDT, and (b) the cationic monomer, DMAPAA-Q. Reproduced with permission from Wu et al. ${ }^{44}$ 
southwest and northwest directions, respectively. Therefore, PBDT is an optically positive LC, ${ }^{42,49,50}$ and POM observation is a facile method for characterizing the alignment of PBDT in aqueous solutions and hydrogels.

Physical gel with macroscopically anisotropic structure from competition of self-assembly and complexation

A significant method for developing hydrogels with macroscopically anisotropic structures uses the reaction-diffusion process. Reaction
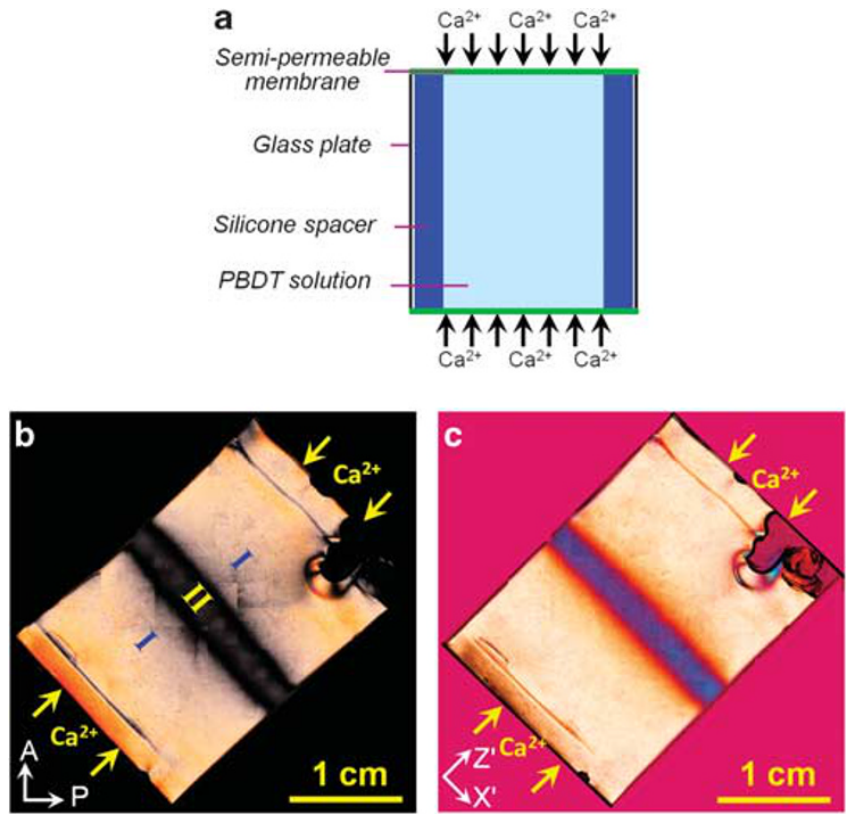

Figure 2 (a) A schematic of the reaction cell used to synthesize physically cross-linked LC hydrogels by dialyzing a PBDT solution in a $\mathrm{CaCl}_{2}$ solution; the dimensions of the cell are $30 \times 20 \times 1 \mathrm{~mm}$ (length $\times$ width $\times$ thickness). (b, c) POM images of the synthesized gel with $C_{P}=1 \mathrm{wt} \%$; crossed polarizers (b), insertion of the tint plate (c). The arrows show the $\mathrm{Ca}^{2+}$ diffusion direction during the synthesis of the gel. The outer and central regions of the gel are denoted as region I and region II, respectively, in (b). Reproduced with permission from Wu et al. ${ }^{45}$ and diffusion compete with each other during this process, which forms intricate spatial or temporal structures. ${ }^{51}$ Dobashi et al. ${ }^{52-55}$ have developed a method to synthesize physical LC hydrogels in a semi-permeable tube by dialyzing aqueous solutions of DNA or curdlan in a multivalent cation solution. The cationic ion diffusion induces molecular orientation and physical gelation of the semi-rigid biomacromolecules to form hydrogels with centimeter-scale anisotropic structures. They have claimed that the biomacromolecules orient along the cationic ions diffusion paths to form a radial structure. ${ }^{52-54}$

We have found that PBDT, which has properties similar to those of biomacromolecules, is also capable of forming macroscopic anisotropic physical gels via a dialysis process. ${ }^{40,45}$ In this process, a $1 \mathrm{wt} \%$ solution of PBDT with a $M \mathrm{w}$ of $2.1 \times 10^{6} \mathrm{~g} \mathrm{~mol}^{-1}$ and a $C_{\mathrm{LC}}{ }^{*}$ of 2 was poured into a mold that consists of a pair of parallel glass plates separated by a $1-\mathrm{mm}$ thick silicone spacer (Figure 2a). ${ }^{45}$ Two opposing sides were left open to permit uniaxial diffusion of multivalent cations into the mold. As $C_{\mathrm{P}}<C_{\mathrm{LC}}{ }^{*}$, the PBDT solution is optically isotropic before the dialysis. After dialysis in $0.5 \mathrm{M} \mathrm{CaCl}_{2}$ solution for 1 day, however, the PBDT solution becomes a gel that shows strong and uniform birefringence at the two outer regions (denoted by region I) and weak birefringence in the narrow central region (denoted by region II). Amorphous linear regions are observed between regions I and II (Figure 2b). After inserting a tint plate, the birefringent image indicates that the PBDT molecules are oriented perpendicular to the diffusion direction in the region I and parallel to the diffusion direction in the region II (Figure 2c). Similar results are observed for high $C_{\mathrm{P}}$. The PBDT alignment in region I of the gel is further confirmed by small angle X-ray scattering measurements. ${ }^{45}$ Our results show that the PBDT molecules align perpendicularly to the $\mathrm{Ca}^{2+}$ diffusion direction, which is opposite to the pattern reported for DNA and curdlan systems by Dobashi et al. ${ }^{51-54}$ However, we have noticed that their previous conclusion about the molecular orientation was not correct, and they have revised their conclusion in a recently published paper. ${ }^{55}$

To track the diffusion-gelation process, we performed in situ observation of the entire gelation process under POM at different dialysis times (Figure 3a). The original solution with $C_{\mathrm{P}}=2 \mathrm{wt} \%=$ $C_{\mathrm{LC}}{ }^{*}$ shows a characteristic schlieren texture. ${ }^{55}$ After $2 \mathrm{~h}$ of dialysis, the regions bordering the $\mathrm{Ca}^{2}+$ diffusion front are gelated with slight
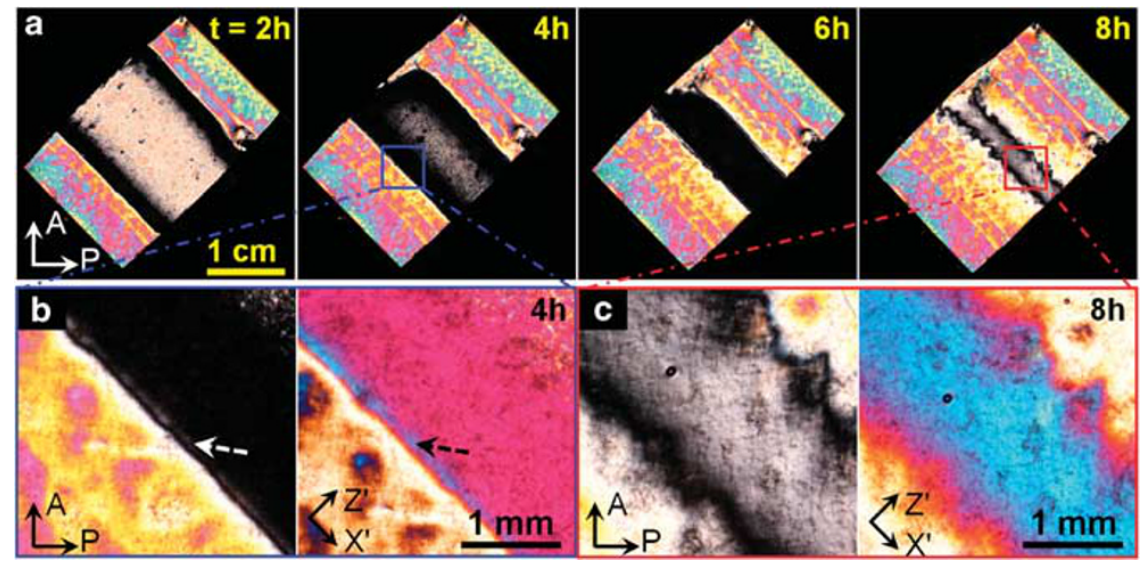

Figure 3 (a) POM images of the gel at different dialysis time; $C_{P}=2$ wt\%. (b, c) Enlarged images of the diffusion front (b) and the central part of the gel (c); the micrographs on the right are observed using a tint plate. The arrows in (b) show the birefringence reversion at the diffusion flux front. The different birefringence colors in region I of (a) observed under POM without a tint plate are caused by varying birefringence that is strong and shifts into the secondorder color rather than by birefringence reversion. Reproduced with permission from Wu et al. ${ }^{45}$ 
turbidity, whereas the central region remains in solution. The gel front line moves toward the center of the cell, and the birefringence of the solution gradually vanishes, indicating decreased $C_{\mathrm{P}}$. It is interesting that at the front of the diffusion flux, a thin birefringent layer $(\sim 100 \mu \mathrm{m})$, shown by the arrow in Figure $3 \mathrm{~b}$, exists that accompanies the progression of the flux toward the center. In this thin layer, the PBDT molecules orient parallel to the diffusion direction, as can be observed using a tint plate. This result indicates that at the flux front, the PBDT alignment shifts from being parallel to the $\mathrm{Ca}^{2+}$ diffusion direction in the solution region to being perpendicular to the $\mathrm{Ca}^{2+}$ diffusion direction in the gelated region. Finally, the flux fronts are terminated when the two gelated regions meet in the central region of the cell. At $8 \mathrm{~h}$, the central region with a width of $\sim 1 \mathrm{~mm}$ is gelated and birefringent, and has PBDT molecules oriented in parallel to the $\mathrm{Ca}^{2}+$ diffusion direction (Figure 3c).

It should be noted that electrostatic complexation with $\mathrm{Ca}^{2+}$ induces PBDT molecules to orient and form mesoscopic fibrous bundles, as is directly observed in the gel synthesized with $C_{\mathrm{P}}=3 \mathrm{wt} \%$ under POM. ${ }^{45}$ Empirically, multivalent ions usually bridge with like-charged rigid or semi-rigid polyelectrolytes to form bundles, ${ }^{56}$ accompanied with the release of monovalent counter ions. ${ }^{57}$ At the $\mathrm{Ca}^{2+}$ ion diffusion front, the PBDT molecules meet with $\mathrm{Ca}^{2+}$ and self-assemble to form fibrous bundles. As the $\mathrm{Ca}^{2+}$ concentration gradient is high at the diffusion front, it is energetically favorable for the highly charged PBDT molecules to orient parallel to the diffusion front, which ensures more complexation. A similar result was reported by Capito et al. and Carvajal et al.,58,59 who dropped aqueous solutions of peptide amphiphiles with positive charges into polyanion solution; the partial charge screening of the peptide amphiphiles led them to self-assemble into fibrous bundles aligned parallel to the liquid-liquid interface.

We have proposed a possible self-assembly mechanism for PBDT/ $\mathrm{Ca}^{2+}$ anisotropic gels (Figure 4). ${ }^{45}$ Before the dialysis, the PBDT solution is macroscopically isotropic. After immersing the reaction cell in a $\mathrm{CaCl}_{2}$ solution, the small $\mathrm{Ca}^{2+}$ ions readily diffuse into the solution of negatively charged PBDT through the semi-permeable membrane, leading to electrostatic complexes along the $\mathrm{Ca}^{2+}$ diffusion front and simultaneous self-assembly of PBDT molecules into mesoscopic fibrous bundles at the diffusion front. Owing to the sharp $\mathrm{Ca}^{2+}$ concentration gradient, the semi-rigid PBDT molecules orient parallel to the diffusion front (perpendicular to the diffusion direction) to form more stable bundles. Meanwhile, the PBDT molecules in the central region diffuse to the $\mathrm{Ca}^{2+}$ flux front due to the local PBDT concentration gradient. As PBDT molecules are transported from the central region of solution to the diffusion flux front, the transverse diffusion (motion) of the semirigid PBDT is severely suppressed by the surrounding rod molecules due to steric constraints, while the longitudinal diffusion is barely influenced. ${ }^{60,61}$ As a result, the PBDT molecules at the diffusion flux front show a favorable orientation that is parallel to the $\mathrm{Ca}^{2+}$ diffusion direction. Finally, the $\mathrm{Ca}^{2+}$ fluxes from the two ends meet and form the LC gel in the narrow central region, with the PBDT molecules aligned parallel to the $\mathrm{Ca}^{2+}$ diffusion direction.

\section{SYNTHESIS OF POLYMERIC HYDROGELS WITH MILLIMETER- SCALE LC STRUCTURES}

Hydrogels with cylindrically symmetric structure by self-assembly of the semi-rigid polyion complex

From synthesizing PBDT physical gels via diffusion processes, we know that controlling the self-assembly direction of PBDT molecules and their complexes is crucial for developing macroscopic LC

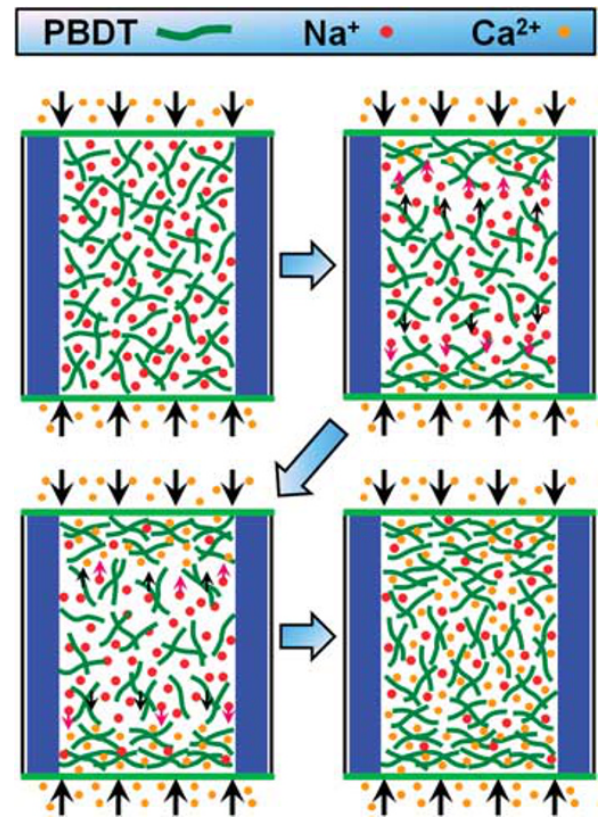

Figure 4 An illustration of complexation-driven anisotropic LC gel formation via dialysis of PBDT solution in $\mathrm{Ca}^{2}+$ solution. Before the $\mathrm{Ca}^{2}+$ diffusion, the PBDT solution is macroscopically isotropic. When the $\mathrm{Ca}^{2}+$ diffuses readily into the anionic PBDT solution, the electrostatic complexation and gelation occur at the diffusion front and the PBDT molecules simultaneously self-assemble into fibrous bundles. Owing to the large $\mathrm{Ca}^{2+}$ concentration gradient, the semi-rigid PBDT molecules orient parallel to the diffusion front (perpendicular to the diffusion direction) to form more stable bundles. Meanwhile, the PBDT molecules in the central region diffuse to the $\mathrm{Ca}^{2}+$ flux front due to the local mobile PBDT concentration gradient. The transverse diffusion (motion) of the semi-rigid PBDT is severely suppressed by steric constrains from the surrounding rod molecules, while the longitudinal diffusion is barely influenced. As a result, the PBDT molecules at the $\mathrm{Ca}^{2}+$ diffusion front show a favorable orientation that is parallel to the $\mathrm{Ca}^{2+}$ diffusion direction. Finally, the two $\mathrm{Ca}^{2+}$ fluxes from the two ends meet and form the LC gel in the narrow central region, with the PBDT molecules parallel to the $\mathrm{Ca}^{2+}$ diffusion direction. The PBDT bundles are not shown here for simplicity. Reproduced with permission from Wu et al. ${ }^{45}$

structures. Our previous work synthesized a hydrogel with anisotropic domains by thermal polymerization of a cationic monomer, $\mathrm{N}$-(3(N,N-dimethylamino)propyl) acrylamide methyl chloride quaternary (DMAPAA-Q), in the presence of a small amount of PBDT functioning as a dopant. ${ }^{38,39}$ The chemical structure of DMAPAA-Q is shown in Scheme 1b. During the polymerization of the isotropic precursor solution, semi-rigid polyion complexes form and selfassemble into $\sim 1 \mathrm{~mm}$ randomly dispersed anisotropic domains. If we could control the self-assembly direction of polyion complexes during the polymerization, it would be possible to develop macroscopically ordered structures in hydrogel.

Our strategy was to polymerize the precursor solution by surround ultraviolet irradiation, as shown in Figure 5a. The glass tube filled with precursor solution was vertically placed in the center of four ultraviolet lamps arranged in a square. The reaction should take place from the outer region to the central, which should control the selfassembly direction. The PBDT used here has an $M w$ of $1.8 \times$ $10^{6} \mathrm{~g} \mathrm{~mol}^{-1}$, much larger than the $8 \times 10^{4} \mathrm{~g} \mathrm{~mol}^{-1}$ that was used in our previous study. ${ }^{38,39}$ The samples are coded as $\mathrm{QP}-C_{\mathrm{Q}}-C_{\mathrm{B}}$ where $C_{\mathrm{Q}}$ is the cationic monomer concentration in $\mathrm{M}$ and $C_{\mathrm{P}}$ is the PBDT concentration in $\mathrm{wt} \%$. All of the samples are synthesized using a 

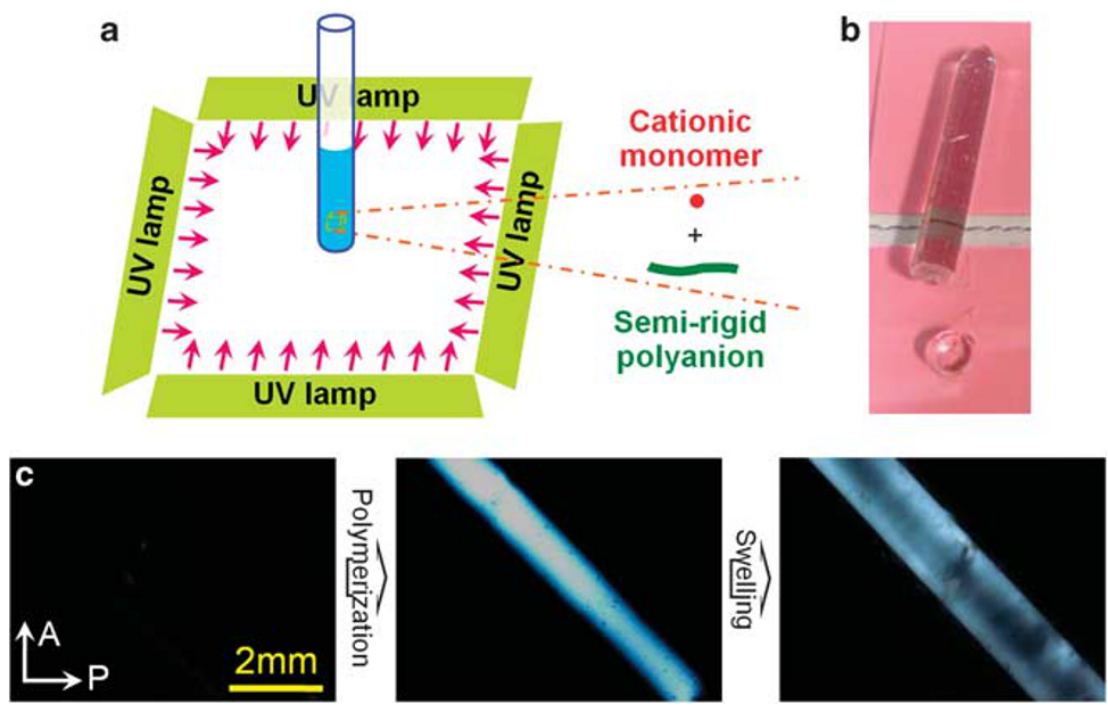

Figure 5 (a) The schematic for an experimental setup to synthesize a cylindrical gel with ordered structures. A glass tube with the precursor solution is vertically placed in the center of four ultraviolet (UV) lamps arranged in a square. (b) The appearance of gel swelled in a 1-M NaCl aqueous solution. The disc piece is cut from the cylinder gel. (c) POM images of the precursor solution in the glass tube, of the as-prepared cylindrical gel, and of the swollen gel (QP-2.5-1). Reproduced with permission from Wu et al. ${ }^{42}$

constant concentration of a chemical cross-linker $(2 \mathrm{~mol} \%)$ and photo-initiator $(0.15 \mathrm{~mol} \%$, relative to the monomer concentration).

The precursor solution has no birefringence. However, the asprepared gel is transparent and exhibits strong birefringence that is maintained after swelling the gel in a $1-\mathrm{M} \mathrm{NaCl}$ solution (Figures $5 \mathrm{~b}, \mathrm{c}$ ). We note that poly(DMAPAA-Q) gels synthesized without PBDT have no birefringence. The PBDT-containing gels synthesized with the anionic monomer 2-acrylamido-2-methylpropanesulfonic acid and neutral one hydroxyethyl acrylate also show no birefringence. Thus, the above oriented structure in PBDT-containing poly(DMAPAA-Q) hydrogels is related to the electrostatic interaction between the polycation and the oppositely charged semi-rigid PBDT that triggers the self-assembly and orientation. Furthermore, the flexible polycation has a much shorter persistence length and entwines with the semi-rigid PBDT; ${ }^{42}$ therefore, the birefringence should mainly be attributed to the oriented PBDTs.

The thin disc gel cut from the bulk cylinder of PBDT-containing poly(DMAPAA-Q) gel showed a huge Maltese cross that did not change when the sample was rotated (Figure 6a). From the birefringent image observed after inserting the tint plate, we know that PBDTs orient radially in the outer region and form a concentric structure in the inner region. The disc gel is then cut into slices parallel to the axis of the cylinder gel to observe the PBDT alignment in the axial plane of gel (Figure $6 \mathrm{~b}$ ). The birefringent image indicates that the PBDT aligns radially in the outer region, perpendicular to the glass surface, whereas it is oriented parallel to the axial direction in the inner region. Therefore, $\mathrm{PBDT}$ is organized in two orthogonal directions in the inner region, partially forming concentric structure and partially aligning with the axial direction. The proposed structure model is shown in Figure 6c.

These gels with complex ordered structures exhibit a sensitive response to external force. The birefringence change of the cylinder and disc gels when compressed in the radial direction is shown in Figure 7. The swollen cylinder gel (QP-2.5-1) shows strong birefringence before compression, indicating that the axial direction alignment of the PBDT predominates when observed from the radial direction. When compression is applied, the central region changes its birefringence color from blue to orange, indicating that the
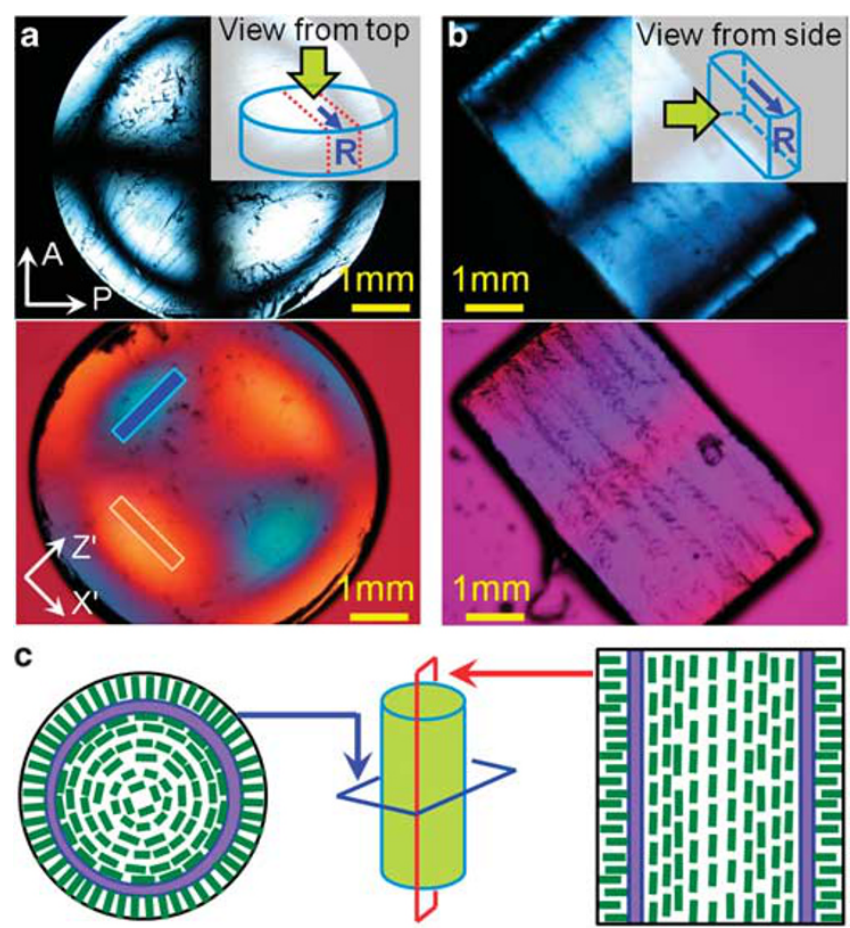

Figure 6 POM images of the QP-2-1 gel after swelling in a $1-\mathrm{M} \mathrm{NaCl}$ aqueous solution and schematics of the PBDT structures in the synthetic gel. Micrographs of the disc gel observed from the top (a) and its central slice observed from the side (b) with crossed polarizers and a tint plate. The inserts show the specimen positioned for the observation and the observation direction; the central slice cut from the disc gel is indicated by the red dotted line. (c) A proposed microstructure of the oriented PBDT in the cross-section and axial plane of polymeric gels. Reproduced with permission from $\mathrm{Wu}$ et al. ${ }^{42}$

preferential axial alignment is overwhelmed by the concentric one (Figures $7 \mathrm{a}, \mathrm{b}$ ). This phenomenon occurs because the compression promotes a concentric orientation of PBDT due to the lateral stretch 
a
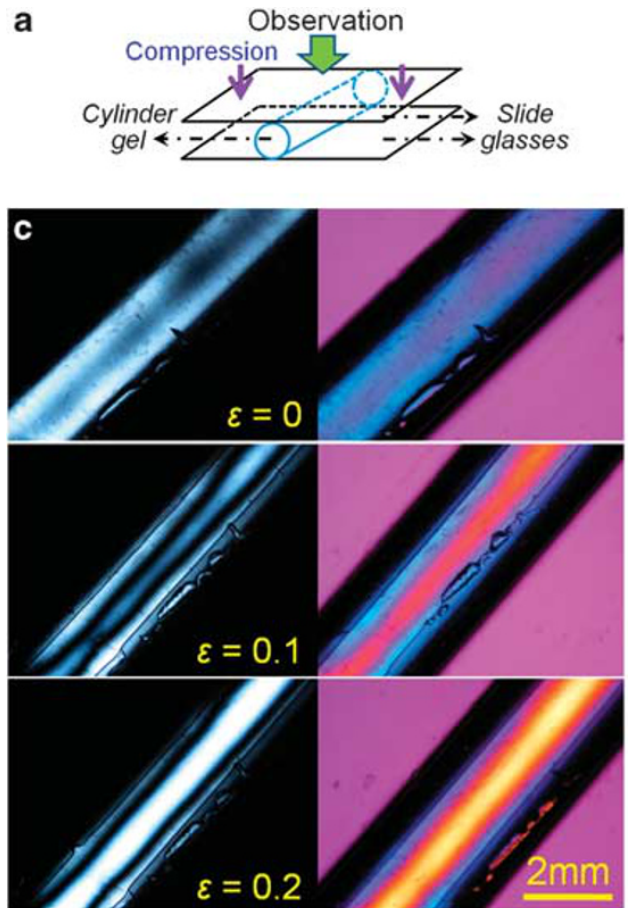

b

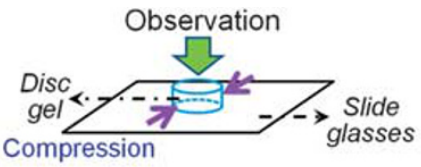

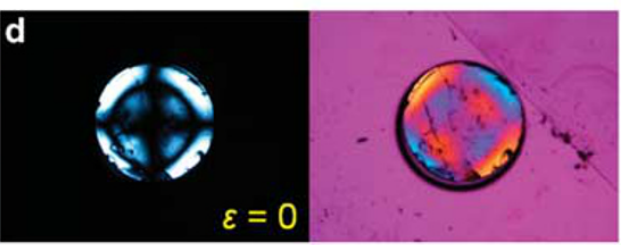
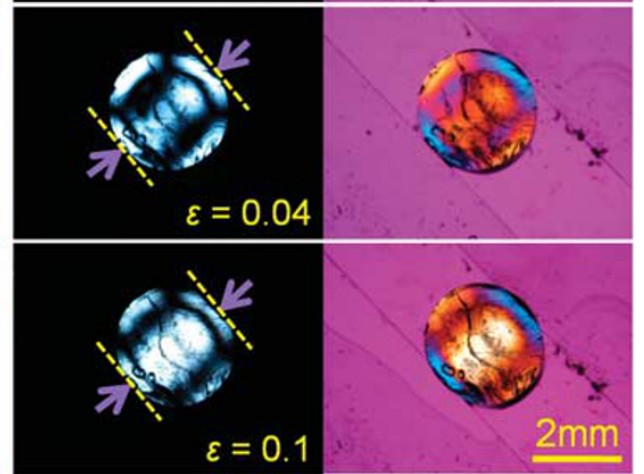

Figure 7 The birefringence changes in the swollen QP-2.5-1 gel when compressed in the radial direction. (a, b) Schematic illustrations of the compression and observations for the cylindrical gel (a) and disc gel (b). (c, d) Crossed polarizing micrographs of QP-2.5-1; the right columns are observed while using a tint plate. The related strain, $\varepsilon$, is noted in the figure. Reproduced with permission from Wu et al. ${ }^{42}$

and decreases the axial orientation due to the decreased thickness. Furthermore, the birefringence change in the disc gels was observed in response to compression from the two sides (Figures $7 \mathrm{c}, \mathrm{d}$ ). When compression is applied, the intensity and range of the blue birefringence in the inner region gradually decrease, whereas the orange intensity and range increase. The compression enhances the PBDT alignment perpendicular to the force direction caused by the lateral stretch; therefore, the concentric alignment in the inner region of the disc gel gradually changes from the circular to elliptical. The results coincide well with our proposed PBDT alignment model for the synthesized gel in Figure 6c.

A mechanism for the formation of cylindrically symmetric structures in hydrogels is proposed in Figure $8 .{ }^{42}$ The precursor solution is isotropic. After photopolymerization, the outer region has higher polymerization kinetics, and the polymerized polycations interact with PBDT to form a semi-rigid polyion complex. Owing to the interfacial interaction of the complex and glass tube, the polyion complexes align perpendicularly with the glass wall to form a radial structure in the outer region. ${ }^{62-64}$ Polymerization in the inner part of the glass tube is retarded due to the significant ultraviolet absorbance of the PBDT, leading to the heterogeneous polymerization, concentration gradient and diffusion of cationic monomers and polycations. ${ }^{42,65,66}$ Monomer diffusion induces the polyion complex to orient perpendicular to the diffusion direction and form the concentric and axial alignments in the inner region (the latter is not shown in Figure 8). The self-organized PBDT structures are frozen by the cross-linking reaction, resulting in polymeric hydrogels with cylindrically symmetric structures up to a macroscopic scale.

Plate hydrogel with cubic-packed giant concentric domains

The cylindrical hydrogels discussed above were synthesized in a glass tube, and the cylindrically symmetric structure may be related to the geometry of the reaction cells. If a rectangular reaction cell is used and other conditions remain constant, is a gel with a macroscopically ordered structure still obtained? Interestingly, the resultant plate hydrogels have cubic-packed concentric domains sandwiched by two homeotropically aligned outer layers. ${ }^{44}$

The precursor solution was poured into reaction cells consisting of a pair of parallel glass plates with 1-mm silicone spacers (Figure 9a). The polymerization was induced by ultraviolet irradiation from the two sides of the reaction cell (Figure 9b). The synthesized plate gel, QP-1.5-1, incorporated $0.5 \mathrm{M} \mathrm{NaCl}$ in the precursor solution and was immersed in a large quantity of pure water for a week. The $\mathrm{NaCl}$ was incorporated during the polymerization to enhance the ionic strength of the solution and suppress phase separation; otherwise, the gel synthesized using $1.5 \mathrm{M}$ DMAPAA-Q is turbid and shows weak birefringence. ${ }^{38,39,41-44}$

After the isotropic precursor solution is polymerized, the asprepared gel shows strong birefringence. However, the birefringence is not homogeneous over the sample, and macroscopic Maltese cross occasionally exists. ${ }^{44}$ It is interesting that after immersing the asprepared gel into pure water, many millimeter-scale Maltese crosses appear in a well-defined cubic packing that does not change when the sample is rotated (Figure 10a, upper image). The birefringent image observed using the tint plate (Figure 10a, lower image) indicates that the cubic-packed Maltese crosses correspond to the concentric orientation of PBDT or its polyion complex, as illustrated in Figure 10c (left illustration).

The structure of the gel was then observed from the lateral direction (the cross-section of the plate gel). When the sample is placed at $45^{\circ}$ against the polarizer or analyzer, it shows strong birefringence in the center region and in the two surface regions close to the substrates. Dark lines parallel to the surfaces are observed between the center region and the surface region (Figure 10b, upper 


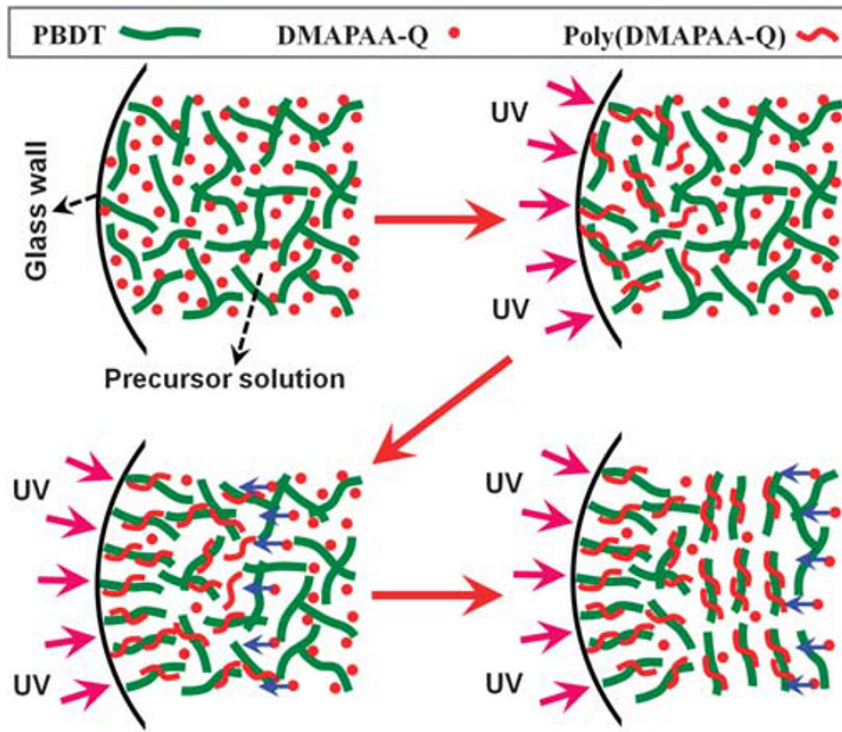

Figure 8 Mechanism for the formation of cylindrically symmetric structures during the photopolymerization of a cationic DMAPAA-Q monomer in the presence of semi-rigid polyanion PBDT serving as a dopant. The precursor solution is optically isotropic because $C_{\mathrm{P}} \ll \mathrm{C}_{\mathrm{LC}}{ }^{*}$. Owing to the strong ultraviolet (UV) absorbance of PBDT, the polymerization starts from the surface of the glass tube, and large amounts of polycations are produced in the outer region and interact with PBDT to form polyion complexes. These semi-rigid complexes favor long-range homeotropic alignment on the glass wall. Simultaneously, a concentration gradient along the radial direction is built up. Thus, the monomers diffuse from the inner region to the outer region. This monomer diffusion induces the polyion complex to orient perpendicular to the diffusion direction and form a concentric structure in the inner region. The various oriented structures are frozen by the subsequent cross-linking reaction. As a result, polymeric hydrogels with macroscopic cylindrical symmetric structures are formed. Reproduced with permission from Wu et al. ${ }^{42}$
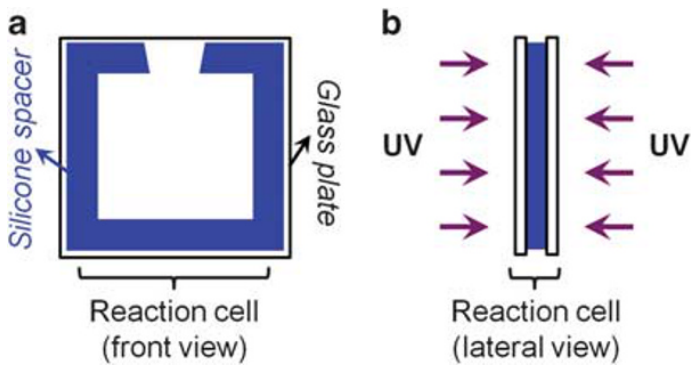

Figure $9 \mathrm{~A}$ scheme for synthesizing a plate gel by photo-initiated polymerization. (a) The front view of the reaction cell, which consists of a pair of glass plates and a silicone spacer. (b) A lateral view of the reaction cell under ultraviolet (UV) irradiation from the two sides. Reproduced with permission from Wu et al. ${ }^{44}$

image). The birefringent image that appears when the tint plate is inserted (lower image of Figure 10b) indicates that the molecules in the surface regions are oriented perpendicularly to the surface, whereas those in the center region are parallel to the surface, as schematically illustrated in Figure 10c (right illustration). In summary, giant concentric cylinders composed of semi-rigid polyion complexes are cubically packed in the inner region of the hydrogel, sandwiched between homeotropically aligned surface layers.
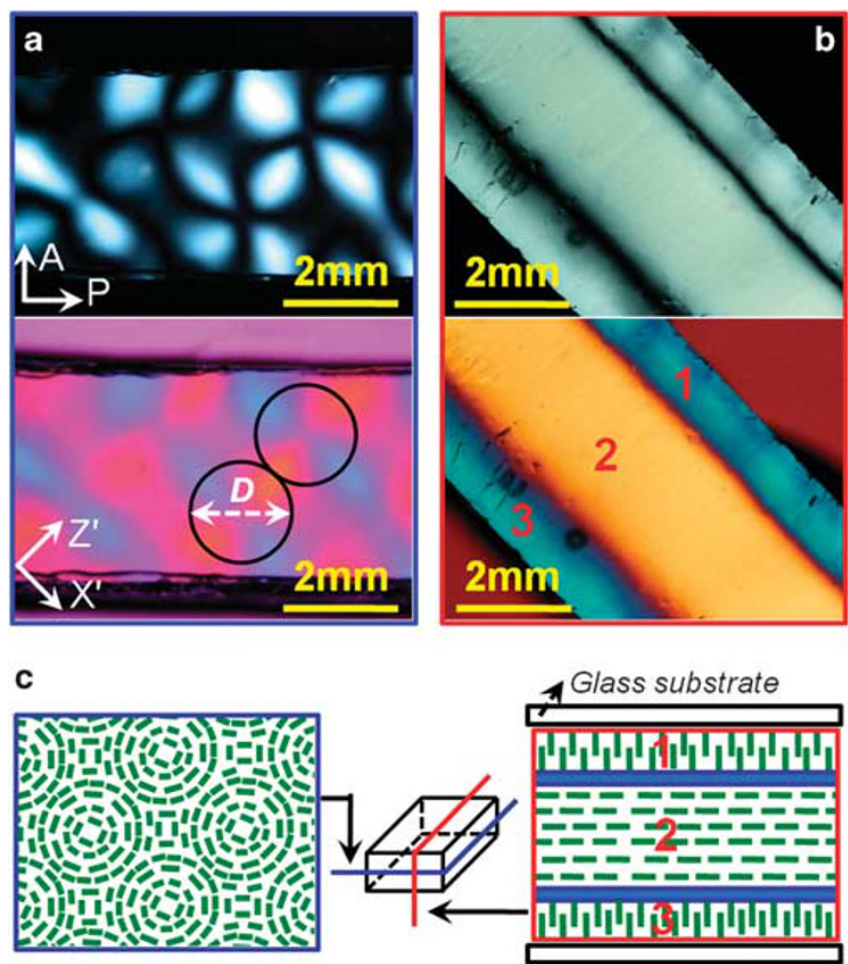

Figure $10(\mathbf{a}, \mathbf{b})$ POM images of the swollen QP-1.5-1 gel observed from the top (a) and the side (b); the upper and lower images are observed without and with a tint plate, respectively. The gel was synthesized by incorporating $0.5-\mathrm{M} \mathrm{NaCl}$ in the precursor solution to suppress the phase separation. The diameter of the concentric domains, $D$, is shown in the lower image of Figure 10a; the three regions (1, 2 and 3$)$ in which the molecules are vertically orientated to each other $(1 \perp 2$ and $2 \perp 3)$ are shown in the lower image of Figure 10b. (c) A simple illustration of the molecular orientation of PBDT and its semi-rigid polyion complex in the gel. Reproduced with permission from Wu et al. ${ }^{44}$

These hydrogels with cubic-packed giant concentric domains can be developed within a large range of $C_{\mathrm{Q}}$ and $C_{\mathrm{P}}$ values. A universal relationship between the diameter of the cylinders, $D$, and the thickness of the swollen gel, $T$, is given by $D=0.5 T$, regardless of the changes in $C_{\mathrm{Q}}$ and $C_{\mathrm{P}} .{ }^{44}$ This result permits us to develop a giant concentric structure with a tunable size in hydorgels. What mechanism should be responsible for forming such complex macroscopic LC structures in hydrogels? Our recent work indicates a non-equilibrium mechanism that will soon be described in a separate paper.

\section{CONCLUDING REMARKS}

Molecular self-assembly is an effective method for creating organized structures in materials, endowing them with robust functions. There has been substantial research into developing functional systems and materials with self-organized structures on a sub-micrometer scale, but macroscopically self-assembled structures are rarely developed in hydrogels. In this short review, we present an overview of our recent studies on synthesizing hydrogels with macroscopically ordered structures based on the self-assembly of a semi-rigid polyanion PBDT. A centimeter-scale anisotropic physical hydrogel has been created by facile diffusion of $\mathrm{Ca}^{2+}$ into PBDT aqueous solution. By contrast, millimeter-scale self-organized structures are developed in polycationic hydrogels based on the self-assembly of polyion complexes. 
In these processes, the PBDT molecules form semi-rigid complexes or bundles through complexation with multivalent $\mathrm{Ca}^{2+}$ cations or polycations; these complexes should be crucial for forming macroscopic self-organized structures. These semi-rigid bundles or fibers of PBDT molecules are much larger than individual molecules and behave as new basic units of self-assembly, as well as the building blocks for macroscopically organized structures. This work should help material science design functional self-organized materials with macroscopic structures.

In addition, the anisotropic hydrogels that have been developed usually have poor mechanical strength, despite being comparable to the conventional single network hydrogels. However, we can greatly improve the strengths of the gels by using the double-network principle, that is, by introducing a second condensed, flexible network into the primary anisotropic gel. ${ }^{46,67-69}$ The hydrogels obtained in this manner possess both self-organized structures and mechanical strength; they should find extensive applications in load-bearing artificial tissues and soft actuators.

\section{ACKNOWLEDGEMENTS}

This research was financially supported by a Grant-in-Aid for Specially Promoted Research (no. 18002002) from the Ministry of Education, Science, Sports and Culture of Japan.

1 Gartner, L. P. \& Hiatt, J. L. Color Textbook of Histology. 2nd ed. (Saunders, Philadelphia, 2001).

2 Sanchez, C., Arribart, H. \& Giraud-Guille, M. M. Biomimetism and bioinspiration as tools for the design of innovative materials and systems. Nat. Mater. 4, 277-288 (2005).

3 Fung, Y. C. Biomechanics: Mechanical Properties of Living Tissues. 2nd ed. (SpringerVerlag Inc., New York, 1993)

4 McGrath, K. \& Kaplan, D. S. Protein-Based Materials (Bikhauser, Boston, 1997).

5 Coppin, C. M. \& Leavis, P. C. Quantitation of liquid-crystalline ordering in F-actin solutions. Biophys. J. 63, 794-807 (1992).

6 Perumal, S., Antipova, O. \& Orgel, J. P. Collagen fibril architecture, domain organization, and triple-helical conformation govern its proteolysis. Proc. Natl. Acad. Sci. USA 105, 2824-2829 (2008)

7 Hirst, L. S., Pynn, R., Bruinsma, R. F. \& Safinya, C. R. Hierarchical self-assembly of actin bundle networks: gels with surface protein skin layers. J. Chem. Phys. 223, 104902 (2005).

8 Zhu, N., Liggitt, D., Liu, Y. \& Debs, R. Systemic gene expression after intravenous DNA delivery into adult mice. Science 261, 209-211 (1993).

9 Kwon, H. J., Kakugo, A., Ura, T., Okajima, T., Tanaka, Y., Furukawa, H., Osada, Y. \& Gong, J. P. Actin network formation by unidirectional polycation diffusion. Langmuir 23, 6257-6262 (2007)

10 Whitesides, G. M. \& Grzybowski, B. Self-assembly at all scales. Science 295, 2418-2421 (2002)

11 Whitesides, G. M., Mathias, J. P. \& Seto, C. T. Molecular self-assembly and nanochemistry: a chemical strategy for the synthesis of nanostructures. Science 254, 1312-1319 (1991)

12 Kato, T. Self-assembly of phase-segregated liquid crystal structures. Science 295 2414-2418 (2002)

13 Kataoka, K., Harada, A. \& Nagasaki, Y. Block copolymer micelles for drug delivery: design, characterization and biological significance. Adv. Drug Deliv. Rev. 47, 113-131 (2001)

14 Li, M.-H., Keller, P., Yang, J. \& Albouy, P.-A. An artificial muscle with lamellar structure based on a nematic troblock copolymer. Adv. Mater. 16, 1922-1925 (2004).

15 Stuart, M. A. C., Huck, W. T. S., Genzer, J., Muller, M., Ober, C., Stamm, M., Sukhorukov, G. B., Szleifer, I., Tsukruk, V. V., Urban, M., Winnik, F., Zauscher, S. Luzinov, I. \& Minko, S. Emerging applications of stimuli-responsive polymer materials. Nat. Mater. 9, 101-113 (2010).

16 Zhang, S. Fabrication of novel biomaterials through molecular self-assembly. Nat Biotechnol 21, 1171-1178 (2003).

17 Hirst, A. R., Escuder, B., Miravet, J. F. \& Smith, D. K. High-tech applications of selfassembling supramolecular nanostructured gel-phase materials: from regenerative medicine to electronic devices. Angew. Chem., Int. Ed. 47, 8002-8018 (2008).

18 Lutolf, M. P. \& Hubbell., J. A. Synthetic biomaterials as instructive extracellular microenvironments for morphogenesis in tissue engineering. Nat. Biotechnol. 23, 47-55 (2005).

19 Zhang, S., Greenfield, M. A., Meta, A., Palmer, L. C., Bitton, R., Mantei, J. R., Aparicio, C., de la Curz, M. O. \& Stupp, S. I. A self-assembly pathway to aligned monodomain gels. Nat. Mater. 9, 594-601 (2010)
20 Tsujii, K., Hayakawa, M., Onda, T. \& Tanaka, T. A novel hybrid material of polymer gels and bilayer membranes. Macromolecules 30, 7397-7402 (1997).

$21 \mathrm{Wu}$, Z. L. \& Gong, J. P. Hydrogels with self-assembling ordered structures and their functions. NPG Asia Mater 3, 57-64 (2011).

22 Osada, Y. \& Matsuda, A. Shape memory in hydrogels. Nature 376, 219 (1995)

23 Kato, T., Mizoshita, N. \& Kishimoto, K. Functional liquid-crystalline assemblies: selforganized soft materials. Angew. Chem., Int. Ed. 45, 38-68 (2006).

24 Chung, H. J. \& Park, T. G. Self-assembled and nanostructured hydrogels for drug delivery and tissue engineering. Nano Today 4, 429-437 (2009).

25 Kang, K., Walish, J. J., Gorishnyy, T. \& Thomas, E. L. Broad-wavelengthrange chemically tunable block-copolymer photonic gels. Nat. Mater. 6, 957-960 (2007).

26 Huang, Z., Lee, H., Lee, E., Kang, S.-K., Man, J.-M. \& Lee, M. Responsive nematic gels from the self-assembly of aqueous nanofibres. Nat. Commun. 2, 459

27 Um, S. H., Lee, J. B., Park, N., Kwon, S. Y., Umbach, C. C. \& Luo, D. Enzymecatalysed assembly of DNA hydrogel. Nat. Mater. 5, 797-801 (2006).

28 Kaneko, T., Yamaoka, K., Gong, J. P. \& Osada, Y. Liquid-crystalline hydrogels. 1. Enhanced effects of incorporation of acrylic acid units on the liquid-crystalline ordering. Macromolecules 33, 412-418 (2000).

29 Kaneko, T., Yamaoka, K., Osada, Y. \& Gong, J. P. Thermoresponsive shrinkage triggered by mesophase transition in liquid crystalline physical hydrogels. Macromolecules 37, 5385-5388 (2004)

30 Kato, T., Hirai, Y., Nakaso, S. \& Moriyama, M. Liquid-crystalline physical gels. Chem Soc. Rev. 36, 1857-1867 (2007).

31 Kleinschmidt, F., Hickl, M., Saalwächter, K., Schmidt, C. \& Finkelmann, H. Lamellar liquid single crystal hydrogels: synthesis and investigation of anisotropic water diffusion and swelling. Macromolecules 38, 9772-9782 (2005).

32 Wall, B. D., Diegelmann, S. R., Zhang, S., Dawidczyk, T. J., Wilson, W. L., Katz, H. E., Mao, H.-Q. \& Tovar, J. D. Aligned macroscopic domains of optoelectronic nanostruc tures prepared via shear-flow sssembly of peptide hydrogels. Adv. Mater. 23, 5009-5014 (2011).

33 Haque, M. A., Kamita, G., Kurokawa, T., Tsujii, K. \& Gong, J. P. Unidirectiona alignment of lamellar bilayer in hydrogel: one-dimensional swelling, anisotropic modulus, and stress/strain tunable structural color. Adv. Mater. 22, 5110-5114 (2010)

34 Haque, M., Kurokawa, T., Kamita, G., Yue, Y. \& Gong, J. P. Rapid and reversible tuning of structural color of a hydrogel over the entire visible spectrum by mechanical stimulation. Chem. Mater. 23, 5200-5207 (2011).

35 Haque, M., Kurokawa, T., Kamita, G. \& Gong, J. P. Lamellar bilayers as reversible sacrificial bonds to toughen hydrogel: hysteresis, self-recovery, fatigue resistance, and crack blunting. Macromolecules 44, 8916-8924 (2011).

36 Funaki, T., Kaneko, T., Yamaoka, K., Ohsedo, Y., Gong, J. P., Osada, Y., Shibasaki, Y. \& Ueda, M. Shear-induced mesophase organization of polyanionic rigid rods in aqueous solution. Langmuir 20, 6518-6520 (2004).

37 Yang, W., Furukawa, H., Shigekura, Y., Shikinaka, K., Osada, Y. \& Gong, J. P. Self-assembling structure in solution of a semirigid polyelectrolyte. Macromolecules 41, 1791-1799 (2008).

38 Shigekura, Y., Chen, Y. M., Furukawa, H., Kaneko, T., Kaneko, D., Osada, Y. \& Gong, J. P. Anisotropic polyion-complex gels via template polymerization. Adv. Mater. 17, 2695-2699 (2005)

39 Shigekura, Y., Furukawa, H., Yang, W., Chen, Y. M., Kaneko, T., Osada, Y. \& Gong, J. P. Anisotropic gelation seeded by a rod-like polyelectrolyte. Macromolecules 40 2477-2485 (2007)

40 Yang, W., Furukawa, H. \& Gong, J. P. Highly extensible double-network gels with selfassembling anisotropic structure. Adv. Mater. 20, 4499-4503 (2008).

41 Wu, Z. L., Furukawa, H., Yang, W. \& Gong, J. P. Mesoscopic network structure of a semi-rigid polyion complex nested in a polyactionic hydrogel. Adv. Mater. 21, 4696-4700 (2009).

42 Wu, Z. L., Kurokawa, T., Liang, S., Furukawa, H. \& Gong, J. P. Hydrogels with cylindrically symmetric structure at macroscopic scale by self-assembly of semi-rigid polyion complex. J. Am. Chem. Soc. 132, 10064-10069 (2010).

$43 \mathrm{Wu}$, Z. L., Kurokawa, T., Liang, S. \& Gong, J. P. Dual network formation in polyelectrolyte hdyrogel via viscoelastic phase separation: role of ionic strength and polymerization kinetics. Macromolecules 43, 8202-8208 (2010).

44 Wu, Z. L., Arifuzzaman, M., Kurokawa, T., Furukawa, H. \& Gong, J. P. Hydrogel with cubic-packed giant concentric domains of semi-rigid polyion complex. Soft Matter 7 , 1884-1889 (2010).

45 Wu, Z. L., Kurokawa, T., Sawada, D., Hu., J., Furukawa, H. \& Gong, J. P. Anisotropic hydrogel from complexation-driven reorientation of semirigid pilyanion at $\mathrm{Ca}^{2+}$ diffusion flux front. Macromolecules 44, 3535-3541 (2011).

46 Wu, Z. L., Sawada, D., Kurokawa, T., Kakugo, A., Yang, W., Furukawa, H. \& Gong, J. P. Strain-induced molecular reorientation and birefringence reversion of a robust, anisotropic double-network hydrogel. Macromolecules 44, 3542-3547 (2011).

47 Vandenberg, E. J., Diveley, W. R., Filar, L. J., Pater, S. R. \& Barth, H. G. The synthesis and solution properties of some rigid-chain, water-soluble polymers: poly[ $N, N^{\prime}$ (sulfo-phenylene)phthalamide]s and poly[ $N, N^{\prime \prime}$-(sulfo-p-phenylene)pyromellitimide $]$ J. Polym. Sci., Part A: Polym. Chem 27, 3745-3757 (1989).

48 Sarkar, N. \& Kershner, L. D. Rigid rod water-soluble polymers. J. Appl. Polym. Chem 27, 393-408 (1989)

49 Murata, K. \& Haraguchi, K. Optical anisotropy in polymer-clay nanocomposite hydroge and its change on uniaxial deformation. J. Mater. Chem 17, 3385-3388 (2007).

50 Demus, D., Goodby, J., Gray, G. W., Spiess, H. W. \& Vill, V. Handbook of Liquid Crystals (Wiley-VCH, Weinheim, 1998). 
51 Grzybowski, B. A., Bishop, K. J. M., Campbell, C. J., Fialkowski, M. \& Smoukov, S. K. Micro- and nanotechnology via reaction-diffusion. Soft Matter 1, 114-128 (2005).

52 Dobashi, T., Nobe, M., Yoshihara, H. \& Konno, A. Liquid crystalline gel with refractive index gradient of curdlan. Langmuir 20, 6530-6534 (2004).

53 Nobe, M., Dobashi, T. \& Yamamoto, T. Dynamics in dialysis process for liquid crystalline gel formation. Langmuir 21, 8155-8160 (2005).

54 Dobashi, T., Furusawa, K., Kita, E., Minamisawa, Y. \& Yamamoto, T. DNA liquidcrystalline gel as adsorbent of carcinogenic agent. Langmuir 23, 1303-1306 (2007).

55 Maki, Y., Ito, K., Hosoya, N., Yoneyama, C., Furusawa, K., Yamamoto, T., Dobashi, T., Sugimoto, Y. \& Wakabayashi, K. Anisotropic structure of calcium-induced alginate gels by optical and small-angle X-ray scattering measurements. Biomacromolecules 12 , 2145-2152 (2011).

56 Butler, J. C., Angelini, T., Tang, J. X. \& Wong, G. C. L. Ion Multivalence and like-charge polyelectrolyte attraction. Phys. Rev. Lett. 91, 028301 (2003).

57 Gummel, J., Cousin, F. \& Boué, F. Counterions release from electrostatic complexes of polyelectrolytes and proteins of opposite charge: a direct measurement. J. Am. Chem. Soc. 129, 5806-5807 (2007).

58 Capito, R. M., Azevedo, H. S., Velichko, Y., Mata, S. A. \& Stupp, S. I. Self-assembly of large and small molecules into hierarchically ordered sacs and membranes. Science 319, 1812-1816 (2008).

59 Carvajal, D., Bitton, R., Mantei, J. R., Velichko, Y. S., Stupp, S. I. \& Shull, K. R. Physical properties of hierarchically ordered self-assembled planar and spherical membranes. Soft Matter 6, 1816-1823 (2010).
60 Szamel, G. Reptation as a dynamic mean-field theory: study of a simple model of rodlike polymers. Phys. Rev. Lett. 70, 3744-3747 (1993).

61 Cush, R., Dorman, D. \& Russo, P. S. Rotational and translational diffusion of tobacco mosaic virus in extended and globular polymer solutions. Macromolecules 37, 9577-9584 (2004).

62 Ujiie, S. \& limura, K. Thermal properties and orientational behavior of a liquidcrystalline ion complex polymer. Macromolecules 25, 3174-3178 (1992).

63 Every, H. A., Van der Ham, L., Picken, S. J. \& Mendes, E. Physical properties of oriented thin films formed by the electrostatic complexation of sulfonated polyaramid. J. Phys. Chem. B. 112, 16403-16408 (2008).

64 Every, H. A., Van der Ham, L., Picken, S. J. \& Mendes, E. Spontaneous homeotropic alignment in films of rigid-flexible polyelectrolyte complexes. Soft Matter 5, 342-345 (2009).

65 Vorflusev, V. \& Kumar, S. Phase-separated composite films for liquid crystal displays. Science 283, 1903-1905 (1999).

66 Désilles, N., Lecamp, L., Lebaudy, P. \& Bunel, C. Gradient structure materials from homogeneous system induced by UV photopolymerization. Polymer 44, 6159-6167 (2003).

67 Gong, J. P., Katsuyama, Y., Kurokawa, T. \& Osada, Y. Double-network hydrogels with extremely high mechanical strength. Adv. Mater. 15, 1155-1158 (2003).

68 Gong, J. P. Why are double network hydrogels so tough? Soft Matter 6, 2583-2590 (2010).

$69 \mathrm{Wu}$, Z. L., Kurokawa, T. \& Gong, J. P. Novel developed systems and techniques based on double-network principle. Bull. Chem. Soc. Jpn. 84, 1295-1311 (2011). 